\title{
Cerebellar foliation via non-uniform cell accumulation caused by fiber-guided migration of granular cells
}

\author{
Hironori TAKEDA***, Yoshitaka KAMEO*,**,***, Takahiro YAMAGUCHI***, \\ Kazunori NAKAJIMA**** and Taiji ADACHI*,****** \\ *Institute for Frontier Life and Medical Sciences, Kyoto University \\ 53 Shogoin-Kawahara-cho, Sakyo, Kyoto 606-8507, Japan \\ E-mail: adachi@infront.kyoto-u.ac.jp \\ **Department of Micro Engineering, Graduate School of Engineering, Kyoto University \\ 53 Shogoin-Kawahara-cho, Sakyo, Kyoto 606-8507, Japan \\ ***Department of Mammalian Regulatory Network, Graduate School of Biostudies, Kyoto University \\ 53 Shogoin-Kawahara-cho, Sakyo, Kyoto 606-8507, Japan \\ ****Department of Anatomy, Keio University School of Medicine \\ 35 Shinanomachi, Shinjuku-ku, Tokyo 160-8582, Japan
}

Received: 7 December 2020; Revised: 23 December 2020; Accepted: 24 December 2020

\begin{abstract}
The cerebellum has a unique morphology characterized by fine folds called folia. During cerebellar morphogenesis, folia formation (foliation) proceeds with granule cell (GC) proliferation in an external granular layer, and subsequent cell migration to an internal granular layer (IGL). GC migration is guided along Bergmann glial (BG) fibers, whose orientation depends on the deformation of cerebellar tissue during folia formation. The aim of this study is to investigate the contribution of the fiber-guided GC migration on folia formation from a mechanical viewpoint. Based on a continuum mechanics model of cerebellar tissue deformation and GC dynamics, we simulated foliation process caused by GC proliferation and migration. By changing migration speeds, we showed that the fiber-guided GC migration caused the non-uniform accumulation of GCs and folia lengthening. Furthermore, the simulation of impaired GC migration under pathological conditions, where GCs did not migrate along BG fibers, revealed that fiber-guided GC migration was necessary for folia lengthening. These simulation results successfully recapitulated the features of physiological and pathological foliation processes and validated the mechanisms that guidance of GC migration by BG fibers causes folia lengthening accompanied by non-uniform IGL. Our computational approach will help us understand biological and physical morphogenesis mechanisms, facilitated by interactions between cellular activities and tissue behaviors.
\end{abstract}

Keywords: Cerebellar morphogenesis, Foliation, Finite element analysis, Continuum mechanics, Cell migration, Tissue growth

\section{Introduction}

The cerebellum has a unique morphology, comprising fine folds called folia. This morphology is associated with cerebellar function, e.g., motor control and cognition, based on evidence that a dysfunctional cerebellum has morphological defects (Sillitoe and Joyner, 2007; Leto et al., 2016). Therefore, to understand formation mechanisms underpinning the functional cerebellum, it is vital to investigate folia formation during cerebellar morphogenesis.

During cerebellar development, cerebellar morphology is formed via foliation mechanisms including folia formation, elongation, and bifurcation. This foliation process is driven by cellular activities including cell proliferation and migration (Corrales et al., 2006; Legue et al., 2016; Leto et al., 2016; Govek et al., 2018; Lejeune et al., 2019). At initial foliation stages, granule cell (GC) precursors proliferate in the external granular layer (EGL) (Sudarov and Joyner, 2007; Legue et al., 2015; Lawton et al., 2019). These GCs extend parallel fibers along a mediolateral axis, and they then migrate inwards along Bergmann glia (BG) fibers (Edmondson and Hatten, 1987; Yacubova and Komuro, 2002a). After migration 
through the EGL, molecular layer (ML), and Purkinje cell layer (PCL), GCs halt, and accumulate to generate an internal granular layer (IGL). Consequently, the IGL becomes thicker around a ridge (lobule), when compared to a folium groove (fissure). Additionally, the height of lobule increases relatively as the fissure position is fixed, leading to the formation of characteristic cerebellar morphology (Sudarov and Joyner, 2007).

Fiber-guided GC migration is believed to be crucial for foliation mechanisms, as impaired GC migration and disorganized BG fibers cause abnormal foliation, including the decrease in lobule height (Men et al., 2015; Ryan et al., 2017; Hughes et al., 2020). A previous study proposed a mechanism of folia lengthening (increase in lobule height), suggesting that fiber-guided migration caused IGL with non-uniform GC accumulation, and subsequent folia outgrowth (Sudarov and Joyner, 2007). This study suggests that BG fibers radiate inward in a fan shape around fissure depending on the tissue deformation. Since the GCs migrate along BG fibers, GCs spread to larger area in the IGL. Conversely, around lobule, BG fibers radiate outward and GCs concentrate to smaller area in the IGL. Therefore, the oriented BG fibers depending on tissue deformation may cause non-uniform GC accumulation and associated tissue growth in the IGL, which cause folia lengthening.

Based on this foliation mechanism, we investigated the contribution of GC migration along BG fibers toward folia lengthening, using computational simulations. For foliation simulation, a continuum mechanics model of tissue deformation and cell migration was developed with reference to a previous cerebral morphogenesis study (Rooij and Kuhl, 2018). Based on our mathematical model, we simulated foliation caused by GC proliferation in the EGL, and then GC migration from the EGL to the IGL. By altering GC migration speeds, we showed that GC migration caused folia lengthening. Furthermore, a simulation of impaired GC migration under pathological conditions showed that fiber orientation was necessary for folia lengthening.

\section{Materials and Methods}

\subsection{Continuum mechanics modeling for cerebellar tissue deformation}

During cerebellar morphogenesis, foliation proceeds with GC dynamics including GC proliferation in the EGL and GC migration to the IGL along BG fibers (Fig. 1A). To investigate foliation mechanisms, we developed a mathematical model of cerebellar tissue deformation, GC proliferation, and migration based on continuum mechanics.

A

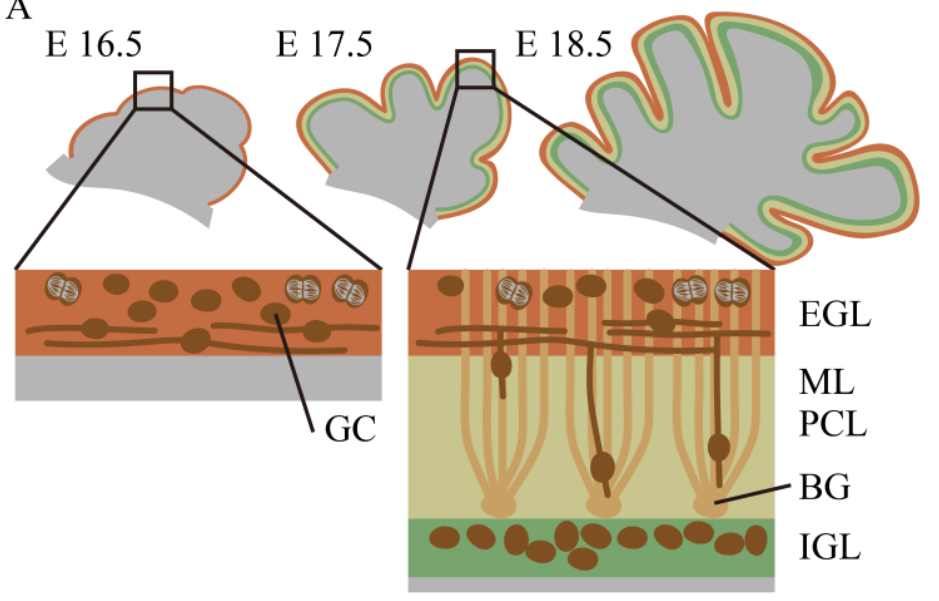

$\mathrm{B}$

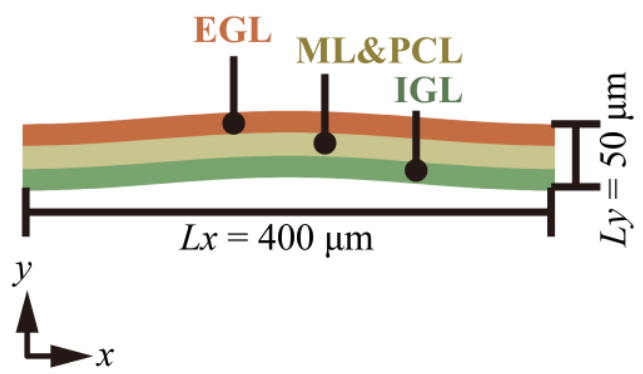

Fig. 1 Modeling cerebellar morphogenesis. (A) Folia formation is accompanied by granule cell (GC) proliferation in the external granular layer (EGL), and subsequent migration into the internal granular layer (IGL) along Bergmann glial (BG) fibers. This folia formation example shows murine cerebellar morphogenesis from embryonic day E16.5-18.5. (B) Initial morphology of cerebellar tissue for finite element analysis consisting of EGL, molecular layer (ML), Purkinje cell layer (PCL) and IGL. The tissue had a length $L_{x}=400 \mu \mathrm{m}$ and a thickness $L_{y}=50 \mu \mathrm{m}$. 
The cerebellar tissue was modeled as a continuum body. We considered points in the body, whose position was denoted by $\boldsymbol{X}$ at the initial state. In the current (deformed) state at time $t$, the position of the same point was moved to $\boldsymbol{x}$ by a deformation map $\chi(\boldsymbol{x}=\chi(\boldsymbol{X}, t))$. Tissue deformation was represented by the deformation gradient tensor $\boldsymbol{F}$ defined as $\boldsymbol{F}=\partial \chi(\boldsymbol{X}, t) / \partial \boldsymbol{X}$. To model volumetric growth, we assumed that the deformation gradient tensor $\boldsymbol{F}$ was multiplicatively decomposed into a growth part $\boldsymbol{F}^{\mathrm{g}}$ and an elastic deformation part $\boldsymbol{F}^{\mathrm{e}}$ (Rodriguez et al., 1994) as:

$$
\boldsymbol{F}=\boldsymbol{F}^{\mathrm{e}} \boldsymbol{F}^{\mathrm{g}} \text {. }
$$

In this study, we assumed isotropic tissue growth because the anisotropy of GC accumulation in IGL was not observed. The deformation gradient $\boldsymbol{F}^{\mathrm{g}}$ can be described by using growth stretch $\theta$ as:

$$
\boldsymbol{F}^{\mathrm{g}}=\theta \boldsymbol{I},
$$

where I was a second order unit tensor. To model tissue growth caused by GC accumulation, we assumed that the volumetric growth depended on cell number density. Thus, the growth stretch was described as a function of cell number density $c(\geq 0)$ in the current state as:

$$
\theta(c)=(1+k c)^{\alpha},
$$

where $k$ and $\alpha$ are constants to represent the relationship between tissue growth and cell number density (Rooij and Kuhl, 2018).

\subsection{Modeling GC proliferation and migration}

To model GC proliferation and migration, the balance equation for cell number density $c$ in the current state was described as:

$$
\frac{\partial c}{\partial t}+c \frac{1}{J} \frac{\partial J}{\partial t}=-\frac{\partial(c \boldsymbol{v})}{\partial \boldsymbol{x}}+D \frac{\partial^{2} c}{\partial \boldsymbol{x}^{2}}+f^{c}
$$

where $J(>0)$ is the Jacobian of the deformation gradient tensor $\boldsymbol{F}$, the vector $\boldsymbol{v}$ is cell migration velocity, $D$ is a diffusion coefficient, and $f^{c}$ is the production rate of cell number density at the current state. The production rate at the initial state $F^{c}$ is represented using $f^{c}$ as $F^{c}=J f^{c}$. The second term on the left side of Eq. (4) shows the effect of tissue deformation on cell number density. The first term on the right side of Eq. (4) shows an advection term for GC migration, the second one shows a diffusion term, and the third one shows a production term for GC proliferation.

Under physiological conditions, where GC migration is guided along BG fibers, GC migration velocity $\boldsymbol{v}$ is described by a directional unit vector $\boldsymbol{a}$ denoting fiber orientation as:

$$
v=|v| a
$$

By assuming current fiber orientation $\boldsymbol{a}$ depends on tissue deformation, thus $\boldsymbol{a}$ is described by the deformation gradient tensor $\boldsymbol{F}$ as:

$$
\boldsymbol{a}=\frac{\boldsymbol{F} \boldsymbol{a}_{0}}{\mid \boldsymbol{F a _ { 0 } |}}
$$

where $\boldsymbol{a}_{0}$ is the initial fiber orientation. To model impaired GC migration under pathological conditions, where GCs do not migrate along BG fibers, we randomly set an angle $\varphi_{\mathrm{r}}\left(-\varphi_{\max } / 2 \leq \varphi_{\mathrm{r}} \leq \varphi_{\max } / 2\right)$ between the fiber direction $\boldsymbol{a}$ and the migration direction $\boldsymbol{v}$.

Through abovementioned modeling, we constructed the mathematical model for GC migration guided by the fiber orientation $\boldsymbol{a}$, which changes from the initial orientation $\boldsymbol{a}_{0}$ depending on tissue deformation $\boldsymbol{F}$, as formulated in Eqs. (5) and (6). The cell number density of GC temporally changes through GC migration and production according to Eq. (4) and affects tissue growth and subsequent tissue deformation, as per Eq. (1-3). 


\subsection{A simulation model for cerebellar foliation}

To investigate foliation mechanisms, we performed numerical simulations based on the above models, using a finite element method. Cerebellar tissue, consisting of EGL, ML, PCL, and IGL, was modeled in a two-dimensional space by assuming plane strain condition (Fig. 1B). Based on experimental observations (Sudarov and Joyner 2007), the tissue had a length $L_{x}=400 \mu \mathrm{m}$ and a thickness $L_{y}=50 \mu \mathrm{m}$, at the initial state $(t=0)$ and each layer had the same thickness. By presupposing the presence of folia at the embryonic day E16.5 (Sudarov and Joyner 2007), the initial tissue shape was slightly curved and followed a sine function having an amplitude of $5 \mu \mathrm{m}$. In numerical simulations, the outof-plane displacement on the lateral surfaces was constrained with fixed top points.

During cerebellar foliation, not all the GCs in the EGL proliferate. We used a cell number density $c$ of the migrating GCs, which do not have a proliferative ability, and assumed that a fixed number of proliferative GCs existed in EGL. Therefore, to model GC production in the EGL, the production rate of cell number density $F^{c}\left(=J f^{c}\right)$ was set to $F^{c}=$ $F_{0}^{c}\left(F_{0}^{c}>0\right)$, and $F^{c}=0$ in the other layers. To model GC migration from the EGL to the IGL, the migration speed $|v|$ was set to $|\boldsymbol{v}|=v_{0}\left(v_{0} \geq 0\right)$ in the EGL, ML and PCL, and $|\boldsymbol{v}|=0$ in the IGL. The migration direction was determined depending on BG fiber orientation as described in Eq. (5). The directional unit vector of fiber orientation $\boldsymbol{a}_{0}$ at the initial state was set to $a_{0 x}=0$ and $a_{0 y}=-1$, where $a_{0 x}$ and $a_{0 y}$ are $x$-and $y$-component of $\boldsymbol{a}_{0}$. These model parameters were defined as listed in Table 1.

Table 1 Model parameters.

\begin{tabular}{c|c|c}
\hline \hline Symbol & Value & Description \\
\hline$L_{x}$ & $400 \mu \mathrm{m}$ & Length of cerebellar tissue \\
$L_{y}$ & $50 \mu \mathrm{m}$ & Thickness of cerebellar tissue \\
$k$ & 1.0 & Constitutive parameter in Eq. (3) \\
$\alpha$ & 0.5 & Constitutive parameter in Eq. (3) \\
$D$ & $5 \mu \mathrm{m}^{2} / \mathrm{h}$ & Diffusion coefficient in Eq. (4) \\
$F_{0}^{c}$ & $0.1 / \mathrm{h}$ & Production rate of cell number density \\
& & in the external granule layer (EGL) \\
$v_{0}$ & $0,5,10 \mu \mathrm{m} / \mathrm{h}$ & Cell migration speed in the external granule layer (EGL), \\
& & molecular layer (ML) and Purkinje cell layer (PCL) \\
$\varphi_{\max }$ & $0, \pi, 3 / 2 \pi$ & Range of random angle $\varphi_{\mathrm{r}}$ \\
\hline
\end{tabular}

\section{Cerebellar morphogenesis; simulation results}

\subsection{Under physiological conditions GC migration causes non-uniform cell accumulation in the IGL, and folia lengthening}

To understand the contribution of GC migration along the BG fibers (oriented based on tissue deformation) to cerebellar morphogenesis, we performed numerical analyses of cerebellar tissue morphogenesis, by altering the cell migration velocity $v_{0}$ as $10 \mu \mathrm{m} / \mathrm{h}$ with reference to the experimental measurement (Yacubova and Komuro, 2002b) and as 5 and $0 \mu \mathrm{m} / \mathrm{h}$ for comparison. Our simulation data showed that GC proliferation in the EGL and their subsequent migration from the EGL to the IGL, caused folia formation (Fig. 2 A-C). The distribution of GC cell number density was temporally changed (Fig. 2A-C; right section). When the migration speed $v_{0}$ was $10 \mu \mathrm{m} / \mathrm{h}$, GC increased in the IGL when compared to other layers, over time $t=20 \mathrm{~h}$ (Fig. 2A). Cell numbers density in the IGL had a non-uniform distribution, and the density were higher around the lobule than the fissure. As the migration speed decreased $\left(v_{0}=\right.$ $5 \mu \mathrm{m} / \mathrm{h}$ ), cell number density decreased in the IGL, but increased in other layers, including the EGL, ML, and PCL, over time $t=20 \mathrm{~h}$ (Fig. 2B). When GCs did not migrate $\left(v_{0}=0 \mu \mathrm{m} / \mathrm{h}\right)$, cell number density became uniformly distributed in the EGL, over time $t=20 \mathrm{~h}$ (Fig. 2C). These data suggested that higher migration speeds caused a non-uniform GC distribution.

To quantitatively analyze the non-uniformity of IGL thickness, temporal changes in difference $d$ in IGL thickness around lobule $d_{1}$ and fissure $d_{\mathrm{f}}$ were plotted (Fig. 2D). Differences in the IGL thickness were larger when GCs migrate $\left(v_{0}=10,5 \mu \mathrm{m} / \mathrm{h}\right)$ than when GCs do not migrate $\left(v_{0}=0 \mu \mathrm{m} / \mathrm{h}\right)$; this result suggested that GC accumulation mediated by GC migration contributed to the formation of an IGL of non-uniform thickness. To analyze the effects of GC migration 
on folia lengthening, temporal changes in the lobule height $h$ was plotted (Fig. 2E). We observed that lobule height increased as migration speed increased. These data suggested that fiber-guided GC migration caused the formation of IGL of non-uniform thickness and folia lengthening, which are characteristic morphogenesis features of the cerebellar tissue.
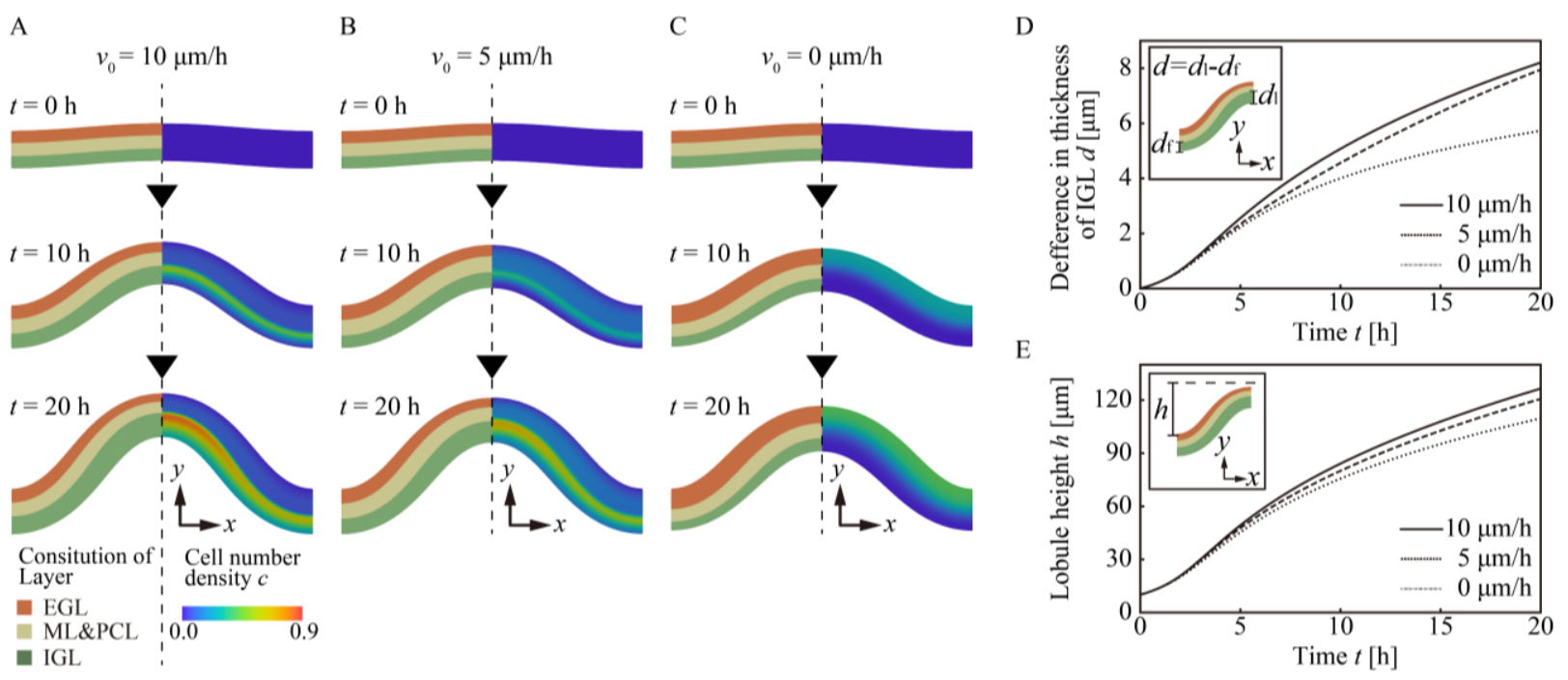

Fig. 2 The effects of fiber-guided migration on granule cell (GC) accumulation and folia length. GCs proliferate in external granular layers (EGLs) and migrate to internal granular layers (IGLs). (A-C) Morphological changes in the cerebellar tissue resulting from GC migration; (A) $v_{0}=10 \mu \mathrm{m} / \mathrm{h},(\mathrm{B}) v_{0}=5 \mu \mathrm{m} / \mathrm{h}$ and (C) $v_{0}=0 \mu \mathrm{m} / \mathrm{h}$ (no migration). The left section of each image shows the cerebellar layer constitution, and the right section shows the distribution of cell number density caused by GC accumulation. (D) Temporal changes in IGL thickness. (E) Temporal changes in lobule height.

\subsection{Impaired GC migration causes defects in folia formation under pathological conditions}

As proposed in a previous study, the orientation of BG fibers, depending on tissue deformation, may be key to the formation of IGL of non-uniform thickness and folia elongation (Sudarov and Joyner, 2007). To investigate the contribution of fiber-guided GC migration, we performed numerical simulations on impaired GC migration, under pathological conditions. Impaired GC migration was modeled, as the migration direction was disturbed by a random angle $\varphi$, ranging from $-\varphi_{\max } / 2$ to $\varphi_{\max } / 2$. We fixed the migration speed at $v_{0}=10 \mu \mathrm{m} / \mathrm{h}$.

The direction field in the EGL, ML and PCL at time $t=20 \mathrm{~h}$ was distributed as shown (Fig $3 \mathrm{~A}-\mathrm{C}$; grey arrows in the enlarged view). When the migration direction generated no disturbance $\left(\varphi_{\max }=0\right)$ corresponding to a physiological condition as previously simulated (Section 3.1), the migration direction spread radially toward the IGL around the fissure, and the migration direction converged toward the IGL around the lobule (Fig. 3A). When the random angle range $\varphi_{\text {max }}$ was set to $\pi$ and $3 \pi / 2$ under pathological conditions, the migration direction was disoriented (Fig. 3B and C; grey arrows in the enlarged view). Cell number density decreased in the IGL, but increased in other layers as random angle range increased (Fig. 3A-C; lower section). To investigate the effects of fiber-guided migration on folia morphology, we plotted temporal changes in difference in IGL thickness between the fissure and lobule $d\left(=d_{1}-d_{\mathrm{f}}\right)$, and lobule height $h$ (Fig. 3D and E). These data indicated that the difference in IGL thickness and the lobule height were smaller as migration randomness increased. Thus, we suggested that an elongated lobule and non-uniform IGL thickness during cerebellar morphogenesis were caused by deformation-dependent GC migration. 

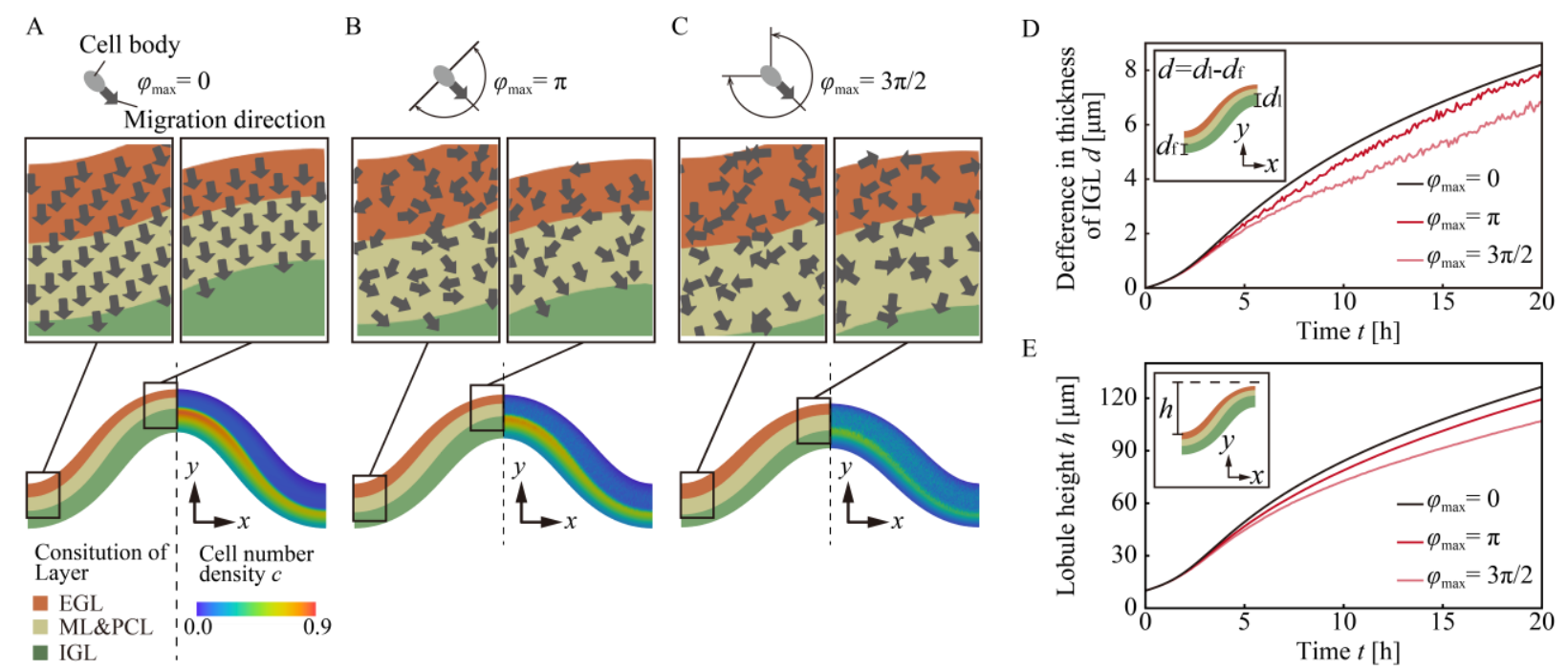

Fig. 3 The effects of impaired granule cell (GC) migration under pathological conditions, where GC migration is not guided on Bergmann glial (BG) fibers, on folia formation. (A-C) The direction field of GC migration over time $t=20 \mathrm{~h}$, as indicated by arrows in the external granule layer (EGL), molecular layer (ML) and Purkinje cell layer (PCL), depending on the random angle $\varphi_{\text {max }}$. Left sections show the cerebellar layer constitution, and right sections show the distribution of cell number density caused by GC accumulation. (D) Temporal changes in IGL thickness. (E) Temporal changes in lobule height.

\section{Discussion}

During cerebellar morphogenesis, the characteristic morphology of the cerebellar tissue is formed via GC migration along BG fibers (Corrales et al., 2006; Legue et al., 2016; Leto et al., 2016; Govek et al., 2018; Lejeune et al., 2019). To investigate the GC mediated foliation, we performed numerical simulations of the cerebellar tissue, driven by GC proliferation and migration. Our simulation results under physiological conditions showed that GC migration from the EGL to the IGL caused elongated folia and a non-uniform IGL which was thicker at the lobule, than the fissure (Fig. 2). Moreover, impaired GC migration under pathological conditions, modeled as random migration directions, resulted in reduced folia lengthening and formation of IGL with uniform thickness (Fig. 3). These results suggested that GC migration along BG fibers was implicated in the formation of characteristic cerebellar morphology, including nonuniform GC accumulation in the IGL, and lengthening folia.

This study validated foliation mechanisms via computer simulation, i.e., folia lengthening accompanied by nonuniform IGL appeared to be caused by GC proliferation and migration along BG fibers (Sudarov and Joyner, 2007). The direction of GC migration corresponding to BG fiber orientation (Fig. 3A) agreed with a previous study (Govek et al., 2018). With impaired and random GC migration, we observed that abnormal foliation, including reduced folia lengthening and formation of IGL with uniform thickness, was caused (Fig. 3B and C). Importantly, these results agree with experimental data; impaired GC migration leads to widely-distributed GCs and a thin IGL (Govek et al., 2018). Therefore, our numerical simulation data successfully recapitulated the features of physiological/pathological foliation processes previously observed in experimental studies (Sudarov and Joyner, 2007; Govek et al., 2018).

We simulated and identified the physical mechanisms that underpin deformation-dependent cell migration and cause non-uniform layer formation during foliation. Such layer formation has been previously explained as the result of biochemically-regulated neuronal migration (D'Arcangelo and Curran, 1998; Sekine et al., 2012; Caffrey et al., 2014; Matsunaga et al., 2017). In addition, the identification of mechanical perspectives is also important in understanding layer formation mechanisms (Lejeune et al., 2016; Engstrom et al., 2018; Lawton et al., 2019). It was previously shown that tissue elastic deformations, such as stretch and compression, during wrinkling caused differences in thickness of multi-layered tissue between lobule and fissure (Tallinen et al., 2014; Holland et al., 2018). In addition to this mechanical 
mechanism, we also proposed a mechanism based on plastic deformation caused by changes in cell number via fiberguided migration. In physiological cerebellar tissue, GC number around lobule is clearly more than around fissure (Sudarov and Joyner, 2007). Because fiber-guided migration is commonly observed during brain morphogenesis such as neuronal migration along radial glial fibers in cerebral development (Borrell and Gotz, 2014; Del Toro et al., 2017; Rahimi-Balaei et al., 2018), our physical mechanism will be important for our increased understanding of layer formation during this process.

In our simulations, we assumed the thickness of each layer at the initial state to be equal (Fig. 1B). The thickness of each layer is considered to affect the number of produced GCs and extent of GC migration. In addition, we assumed the initial folia height as a specific value, which can influence the magnitude of deformation. However, because these assumptions affected only the quantitative results, the setting of the initial tissue state does not affect the qualitative results for the proposed foliation mechanism, where the folia elongate via non-uniform GC accumulation caused by fiberguided GC migration. For the simulation of a specific condition, a further analysis is required. To apply this model to a three-dimensional analysis for the entire cerebellum, the effects of a cerebellar tissue curvature on foliation must be investigated in the future.

The foliation mechanism investigated in this research presupposed the existence of the initial fissure, even though initial foliation was important for the determination of cerebellar morphology. To comprehensively understand the foliation, it is important to investigate the formation of an initial fissure by considering other mechanisms, such as fluidlike tissue behavior resulting from cell migration in the EGL (Engstrom et al., 2018; Lawton et al., 2019). Moreover, folia bifurcation in parallel with folia lengthening is important for the study of cerebellar morphogenesis. To investigate folia bifurcation, other mechanical factors that cause bifurcation must be considered, such as lobule-lobule contact. In addition to mechanisms implicated in the formation of characteristic tissue morphology, further developments may reveal mechanisms for establishment of the physiological function by considering the tissue morphology associates with its function (Sillitoe and Joyner, 2007; White and Sillitoe, 2013; Leto et al., 2016).

\section{Conclusion}

In this study, we constructed the computational model for cerebellar foliation caused by fiber-guided cell migration. Through finite element analysis, we successfully recapitulated the features of foliation processes and validated foliation mechanisms, i.e., folia lengthening accompanied by non-uniform IGL appeared to be caused by GC proliferation and migration along BG fibers. Our computational approach has provided insights on the biological and physical mechanisms that characterize cerebellar morphogenesis, and may facilitate a greater understanding of interactions between cellular activities and tissue behaviors.

\section{Acknowledgement}

This work was supported by Grant-in-Aid for Scientific Research on Innovative Areas (JP16H06486, JP16H06482), by Grant-in-Aid for Scientific Research(S) (JP20H05688), and by Grant-in-Aid for JSPS fellows (19J14843) from Japan Society for the Promotion of Science KAKENHI.

\section{References}

Borrell, V. and Götz, M., Role of radial glial cells in cerebral cortex folding, Current Opinion in Neurobiology, Vol. 27, (2014), DOI: 10.1016/j.conb.2014.02.007

Caffrey, J. R., Hughes, B. D., Britto, J. M. and Landman, K. A., An in silico agent-based model demonstrates Reelin function in directing lamination of neurons during cortical development, PLoS One, Vol. 9, No. 10 (2014), DOI: 10.1371/journal.pone. 0110415

Corrales, J. D., Blaess, S., Mahoney, E. M. and Joyner, A. L., The level of sonic hedgehog signaling regulates the complexity of cerebellar foliation, Development, Vol. 133, No. 9 (2006), DOI: 10.1242/dev.02351

D'Arcangelo, G. and Curran, T., Reeler: new tales on an old mutant mouse, Bioessays, Vol. 20, No. 3 (1998), DOI: 10.1002/(SICI)1521-1878(199803)20:3<235::AID-BIES7>3.0.CO;2-Q

Del Toro, D., Ruff, T., Cederfjall, E., Villalba, A., Seyit-Bremer, G., Borrell, V. and Klein. R., Regulation of Cerebral 
Takeda, Kameo, Yamaguchi, Nakajima and Adachi, Journal of Biomechanical Science and Engineering, Vol.16, No.1 (2021)

Cortex Folding by Controlling Neuronal Migration via FLRT Adhesion Molecules, Cell, Vol. 169, No.4 (2017), DOI: $10.1016 /$ j.cell.2017.04.012

Edmondson, J. C. and Hatten, M. E., Glial-guided granule neuron migration in vitro: a high-resolution time-lapse video microscopic study, The Journal of Neuroscience, Vol. 7, No.6 (1987), DOI: 10.1523/JNEUROSCI.07-0601928.1987

Engstrom, T. A., Zhang, T., Lawton, A. K., Joyner, A. L. and Schwarz. J. M., Buckling without Bending: A New Paradigm in Morphogenesis, Physical Review X, Vol. 8, No.4 (2018), DOI: 10.1103/PhysRevX.8.041053

Govek, E. E., Wu, Z., Acehan, D., Molina, H., Rivera, K., Zhu, X., Fang, Y., Tessier-Lavigne, M. and Hatten, M. E., Cdc42 Regulates Neuronal Polarity during Cerebellar Axon Formation and Glial-Guided Migration, iScience, Vol. 1, No.23 (2018), DOI: 10.1016/j.isci.2018.01.004

Holland, M., Budday, S., Goriely, A., and Kuhl. E., Symmetry Breaking in Wrinkling Patterns: Gyri Are Universally Thicker than Sulci, Physical Review Letter, Vol. 121, No.22 (2018), DOI: 10.1103/PhysRevLett.121.228002

Hughes, L. J., Park, R., Lee, M. J., Terry, B. K., Lee, D. J., Kim, H., Cho, S. H. and Kim, S., Yap/Taz are required for establishing the cerebellar radial glia scaffold and proper foliation, Developmental Biology, Vol. 457, No.1 (2020), DOI: 10.1016/j.ydbio.2019.10.002

Lawton, A. K., Engstrom, T., Rohrbach, D., Omura, M., Turnbull, D. H., Mamou, J., Zhang, T., Schwarz, J. M. and Joyner, A. L., Cerebellar folding is initiated by mechanical constraints on a fluid-like layer without a cellular prepattern, Elife, Vol. 8, (2019), DOI: 10.7554/eLife.45019

Legué, E., Gottshall, J. L., Jaumouillé, E., Roselló-Díez, A., Shi, W., Barraza, L. H., Washington, S., Grant, R. L. and Joyner, A. L., Differential timing of granule cell production during cerebellum development underlies generation of the foliation pattern, Neural Development, Vol. 11, (2016), DOI: 10.1186/s13064-016-0072-z

Legué, E., Riedel, E. and Joyner, A. L., Clonal analysis reveals granule cell behaviors and compartmentalization that determine the folded morphology of the cerebellum, Development, Vol. 142, No.9 (2015), DOI: 10.1242/dev.120287

Lejeune, E., Dortdivanlioglu, B. Kuhl, E. and Linder, C., Understanding the mechanical link between oriented cell division and cerebellar morphogenesis, Soft Matter, Vol. 15, No.10 (2019), DOI: 10.1039/C8SM02231C

Lejeune, E., Javili, A., Weickenmeier, J., Kuhl, E. and Linder, C., Tri-layer wrinkling as a mechanism for anchoring center initiation in the developing cerebellum, Soft Matter, Vol. 12, No.25 (2016), DOI: 10.1039/C6SM00526H

Leto, K., Arancillo, M., Becker, E. B., Buffo, A., Chiang, C., Ding, B., Dobyns, W. B., Dusart, I., Haldipur, P., Hatten, M. E., Hoshino, M., Joyner, A. L., Kano, M., Kilpatrick, D. L., Koibuchi, N., Marino, S., Martinez, S., Millen, K. J., Millner, T. O., Miyata, T., Parmigiani, E., Schilling, K., Sekerkova, G., Sillitoe, R. V., Sotelo, C., Uesaka, N., Wefers, A., Wingate, R. J. and Hawkes, R., Consensus Paper: Cerebellar Development, Cerebellum, Vol. 15, No.6 (2016), DOI: 10.1007/s12311-015-0724-2

Matsunaga, Y., Noda, M., Murakawa, H., Hayashi, K., Nagasaka, A., Inoue, S., Miyata, T., Miura, T., Kubo, K. I. and Nakajima, K., Reelin transiently promotes N-cadherin-dependent neuronal adhesion during mouse cortical development, Proceedings of the National Academy of Sciences, Vol. 114, No.8 (2017), DOI: 10.1073/pnas.1615215114

Men, Y., Zhang, A., Li, H., Jin, Y., Sun, X., Li, H. and Gao, J., LKB1 Regulates Cerebellar Development by Controlling Sonic Hedgehog-mediated Granule Cell Precursor Proliferation and Granule Cell Migration, Science Reports, Vol. 5, No.1 (2015), DOI: 10.1038/srep16232

Rahimi-Balaei, M., Bergen, H., Kong, J. and Marzban, H., Neuronal Migration During Development of the Cerebellum, Frontiers in Cellular Neuroscience, Vol. 12, (2018), DOI: 10.3389/fncel.2018.00484

Rodriguez, E. K., Hoger, A. and McCulloch, A. D., Stress-dependent finite growth in soft elastic tissues, Journal of Biomechanics, Vol. 27, No. 4 (1994), DOI: 10.1016/0021-9290(94)90021-3

Rooij, R., and Kuhl, E., A physical multifield model predicts the development of volume and structure in the human brain, Journal of the Mechanics and Physics of Solids, Vol. 112, (2018), DOI: 10.1016/j.jmps.2017.12.011

Ryan, K. E., Kim, P. S., Fleming, J. T., Brignola, E., Cheng, F. Y., Litingtung, Y. and Chiang. C., Lkb1 regulates granule cell migration and cortical folding of the cerebellar cortex, Developmental Biology, Vol. 432, No. 1 (2017), DOI: 10.1016/j.ydbio.2017.09.036

Sekine, K., Kawauchi, T., Kubo, K., Honda, T., Herz, J., Hattori, M., Kinashi, T. and Nakajima, K., Reelin controls neuronal positioning by promoting cell-matrix adhesion via inside-out activation of integrin alpha5beta1, Neuron, Vol. 76, No.2 (2012), DOI: 10.1016/j.neuron.2012.07.020 
Takeda, Kameo, Yamaguchi, Nakajima and Adachi, Journal of Biomechanical Science and Engineering, Vol.16, No.1 (2021)

Sillitoe, R. V., and Joyner, A. L., Morphology, molecular codes, and circuitry produce the three-dimensional complexity of the cerebellum, Annual Review of Cell and Developmental Biology, Vol. 23, (2007), DOI: 10.1146/annurev.cellbio.23.090506.123237

Sudarov, A., and Joyner, A. L., Cerebellum morphogenesis: the foliation pattern is orchestrated by multi-cellular anchoring centers, Neural Development, Vol. 2, No. 1 (2007), DOI: 10.1186/1749-8104-2-26

Tallinen, T., Chung, J. Y., Biggins, J. S. and Mahadevan, L., Gyrification from constrained cortical expansion, Proceedings of the National Academy of Sciences, Vol. 111, No. 35 (2014), DOI: 10.1073/pnas.1406015111

White, J. J., and Sillitoe, R. V., Development of the cerebellum: from gene expression patterns to circuit maps, Wiley Interdisciplinary Reviews-Developmental Biology, Vol. 2, No. 1 (2013), DOI: 10.1002/wdev.65

Yacubova, E., and Komuro, H., Cellular and molecular mechanisms of cerebellar granule cell migration, Cell Biochemistry and Biophysics, Vol. 37, No. 3 (2002a), DOI: 10.1385/CBB:37:3:213

Yacubova, E., and Komuro, H., Stage-specific control of neuronal migration by somatostatin, Nature, Vol. 415, (2002b), DOI: $10.1038 / 415077 \mathrm{a}$ 